\title{
VIRTUAL PHOTOPRODUCTION OF PROTONS ON HYDROGEN
}

\author{
H. ACKERMANN, T. AZEMOON, W. GABRIEL, D. LÜKE * and G. SPECHT \\ Deutsches Elektronen-Synchrotron DESY, Hamburg \\ E. GANSSAUGE \\ Universität Marburg \\ F. JANATA, H.D. MERTIENS, H.D. REICH and D. SCHMIDT \\ Gesamthochschule Wuppertal
}

Received 11 October 1976

In this paper data on the inclusive reaction $\mathrm{e}^{-} \mathrm{p} \rightarrow \mathrm{e}^{-} \mathrm{p}+$ anything as obtained in a coincidence experiment at DESY are presented. The invariant cross section is given as a function of $q^{2}, W, x, p_{\perp}^{2}$ and $\phi$ in the following kinematical region:

$$
\begin{aligned}
-0.64 & <q^{2}<-0.08 \mathrm{GeV}^{2}, \\
1.96 & <W<2.80 \mathrm{GeV}, \\
0 & <x<1, \\
0 & <p_{\perp}^{2}<0.29 \mathrm{GeV}^{2}, \\
0^{\circ} & <\phi<360^{\circ} .
\end{aligned}
$$

The inclusive spectra are compared with the results from photoproduction and other electroproduction experiments.

\section{Introduction}

This report presents the inclusive proton distributions obtained in an electroproduction experiment $\mathrm{e}^{-} \mathrm{p} \rightarrow \mathrm{e}^{-} \mathrm{p}+$ anything ${ }^{\star \star}$. The differential cross section was measured by detecting coincidences between the scattered electron and outgoing proton, and its dependence on $q^{2}, W, x, p_{\perp}^{2}$ and $\phi$ which are defined below was stu. died. If the four momenta of the primary electron, the scattered electron, the virtual photon, the target proton and the outgoing proton are indicated by $e, e^{\prime}, \gamma_{\mathrm{v}}, p$

* Now at SLAC, Stanford, Calif. 94305, USA.

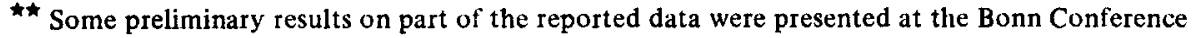
[1], where too high a normalization was used. 
and $p^{\prime}$ respectively, then:

$q^{2}=\left(e-e^{\prime}\right)^{2}$ is the square of the mass of the virtual photon.

$W=\sqrt{\left(\gamma_{\mathrm{v}}+p\right)^{2}}$ is the c.m. energy of the virtual photon and the target proton.

$p_{\perp}^{2}$ is the square of the transverse momentum of the outgoing proton with respect to the direction of the photon.

$x=p_{\|}^{*} / p_{\max }^{*}$ is the Feynman longitudinal variable, where $p_{\|}^{*}$ is the longitudinal momentum of the outgoing proton with respect to the direction of the photon, and $p_{\max }^{*}$ is the maximum momentum of the outgoing proton at the observed value of $W$, which is attained in the reaction $\gamma_{v} p \rightarrow \pi^{0} p$. Both momenta refer to the c.m.s. of the photon and the target proton.

$\phi$ is the azimuthal angle of the outgoing proton which is the angle between the polarization plane, subtended by $e$ and $e^{\prime}$, and the production plane, subtended by $\gamma_{v}$ and $\boldsymbol{p}^{\prime}$.

Treating electroproduction as photoproduction by virtual photons the cross section for the reaction $e^{-} p \rightarrow e^{-} p+$ anything is written [2], assuming one-photon exchange as

$$
\frac{\mathrm{d}^{5} \sigma}{\mathrm{d} q^{2} \mathrm{~d} W \mathrm{~d} p}=2 \pi \Gamma \frac{\mathrm{d}^{3} \sigma}{\mathrm{d} p}
$$

where $\mathrm{d}^{3} \sigma / d p$ is the cross section for $\gamma_{\mathrm{v}} \mathrm{p} \rightarrow \mathrm{p}+$ anything and $\Gamma$, the "flux' of the virtual photons, is given by

$$
\Gamma=\frac{\alpha}{4(2 \pi)^{2}} \frac{2 W}{E_{0}^{2} M_{\mathrm{p}}^{2}\left|q^{2}\right|} \frac{W^{2}-M_{\mathrm{p}}^{2}}{1-\epsilon} .
$$

Here $E_{0}$ is the energy of the primary electron in the lab system, $M_{\mathrm{p}}$ is the mass of proton and $\epsilon$ is the degree of the transverse polarization of the photon:

$$
\epsilon=\left(1+2 \frac{q^{2}}{\left|q^{2}\right|} \operatorname{tg}^{2} \frac{1}{2} \theta_{\mathrm{ee}^{\prime}}\right)^{-1}
$$

where $\theta_{\mathrm{ee}}$ is the scattering angle of the electron.

In this paper the differential cross section for the virtual photoproduction is given in the Lorentz invariant form normalized with the total cross section, $\left(E / \sigma_{\text {tot }}\right)\left(\mathrm{d}^{3} \sigma /\right.$ $\mathrm{d} p)$ ( $E$ is the energy of the detected proton). In the framework of one-photon exchange the above cross section can be parametrized as

$$
\frac{E}{\sigma_{\text {tot }}} \frac{\mathrm{d}^{3} \sigma}{\mathrm{d} \boldsymbol{p}} \equiv f=f_{\mathrm{u}}+\epsilon f_{\mathrm{L}}+\epsilon f_{\mathrm{p}} \cos 2 \phi+\sqrt{2 \epsilon(\epsilon+1)} f_{\mathrm{I}} \cos \phi .
$$

The subscripted functions depend on $q^{2}, W, x$ and $p_{\perp}^{2}$ only and the four terms appearing on the right-hand side of eq. (1) are the contributions from the unpolarized transverse virtual photons, the longitudinal photons, the transverse linear polariza- 

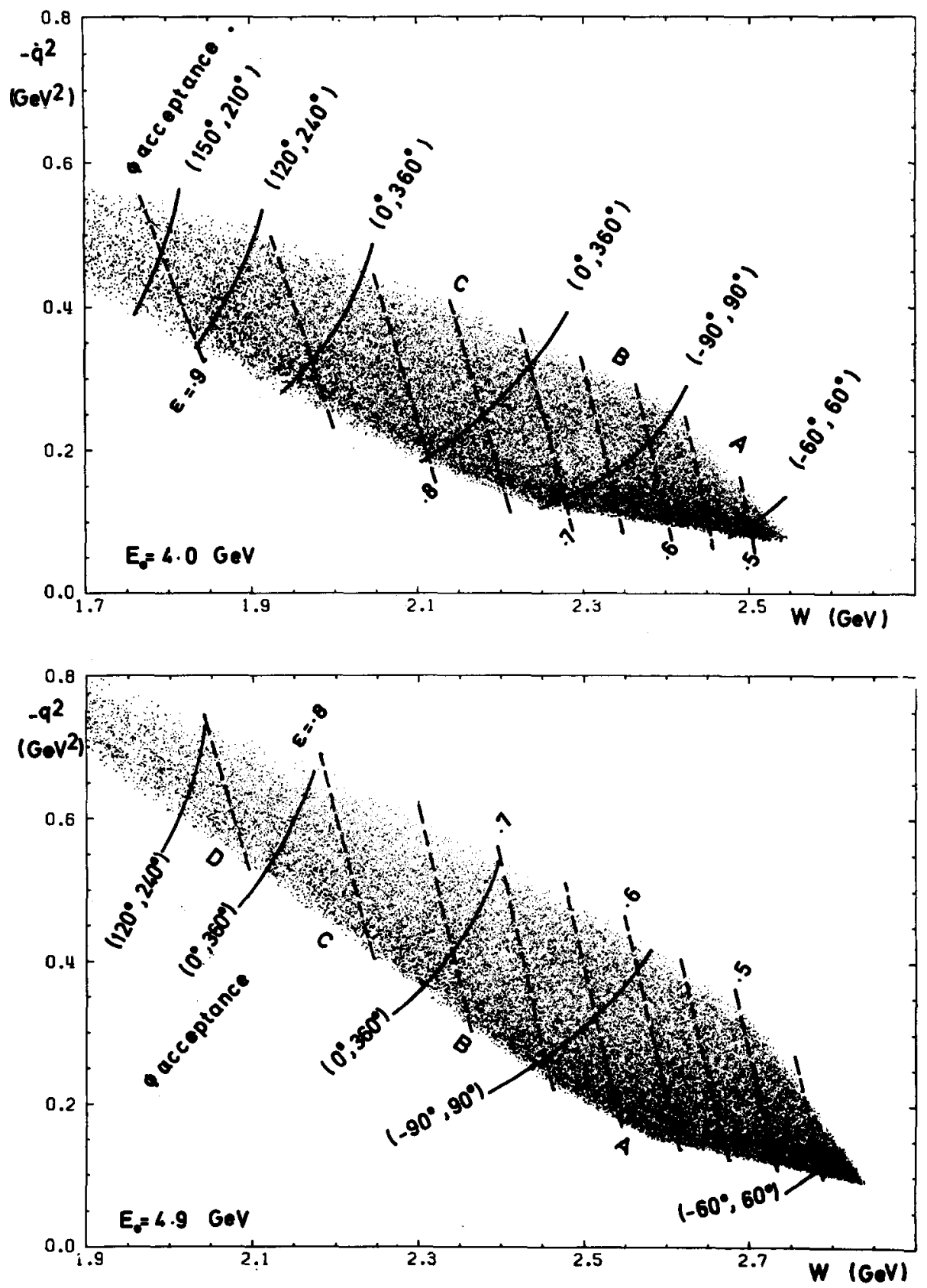

Fig. 1. Distribution of the experimental data in the $q^{2}-W$ plane. (a) For $E_{0}=4.0 \mathrm{GeV}$. (b) For $E_{0}=4.9 \mathrm{GeV}$. 
tion of the photon and the interference between the transverse and longitudinal com. ponents. The values of $\sigma_{\text {tot }}$, which depend on $q^{2}$ and $W$ only, were taken from ref. $[3]$.

\section{Data analysis and corrections}

The experimental details about the spectrometers which detected the scattered electron and the outgoing proton can be found in ref. [4]. The separation of protons, kaons and pions by means of a Čerenkov counter and a time-of-flight measurement system has been described in ref. [5].

In order to cover a large range in $q^{2}$ and $W$, the data were obtained at two different energies of the primary electron, $E_{0}=4.0$ and $4.9 \mathrm{GeV}$. Figs. $1 \mathrm{a}$ and $1 \mathrm{~b}$ show the distribution of the data in $W$ and $q^{2}$ for the two energies. The solid lines indicate the range in the azimuthal angle $\phi$ covered by the apparatus and the broken lines correspond to the constant values of $\epsilon$.

The cross sections were corrected for the background and contamination due to unidentified kaons and for the loss of proton events outside the cuts in the time of flight spectra [6]. These corrections varied between 1 and 20\% depending on the momentum of the proton. Corrections were also applied for efficiency loss in the trigger counters and the electron Cerenkov and shower counters (6\%), for the strong interaction of the protons ( $2 \%$ ) and for the loss in the automatic data analysis procedure. The uncertainties in all these corrections including that in the intensity of the primary beam add up to an overall systematic error of less than $4 \%$ which is not included in the errors given in this report. Neither have the radiative corrections been taken into account, which are of the order of a few per cent.

\section{Results}

Due to strong dependence of the acceptance in the azimuthal angle $\phi$ on the variables $W$ and $q^{2}$ (figs. 1 a and $1 \mathrm{~b}$ ), the data were analysed in four distinct kinematical regions with the following limits on $\phi$ :

$$
\begin{array}{ll}
\text { Region A }-60^{\circ}<\phi<60^{\circ}, & \text { Region C } 0^{\circ}<\phi<360^{\circ}, \\
\text { Region B }-90^{\circ}<\phi<90^{\circ}, & \text { Region D } 120^{\circ}<\phi<240^{\circ} .
\end{array}
$$

In region $C$, were full acceptance in $\phi$ was achieved, the three components $f_{\mathrm{u}}+\epsilon f_{\mathrm{L}}$, $f_{\mathrm{p}}$ and $f_{\mathrm{I}}$ were separated through the azimuthal dependence of the invariant cross section $f$, and their dependence on the variables $q^{2}, W, x$ and $p_{\perp}^{2}$ was investigated. In all cases the first component was the dominant one with the other two contributing less than $10 \%$ to the measured cross sections (figs. $3 a, b$ and $5 a, b$ ). In the remaining regions, the cross sections were averaged over the accepted $\phi$ range. Eq. 
Table 1

Values of $\alpha$ and $\beta$ for different kinematical regions

\begin{tabular}{llllr}
\hline Region & A & B & C & D \\
\hline$\alpha$ & $0.19-0.27$ & 0 & 0 & 0.34 \\
$\beta$ & $0.95-10.22$ & $0.91-1.02$ & 0 & -1.44 \\
\hline
\end{tabular}

(1) averaged over this $\phi$ range gives

$$
\begin{aligned}
\bar{f} & =\frac{1}{\phi_{\max }-\phi_{\min }} \int_{\phi_{\min }}^{\phi_{\max }} f \mathrm{~d} \phi=f_{\mathrm{u}}+\epsilon f_{\mathrm{L}}+\alpha\left(\phi_{\min }, \phi_{\max }, \epsilon\right) f_{\mathrm{p}} \\
& +\beta\left(\phi_{\min }, \phi_{\max }, \epsilon\right) f_{\mathrm{I}} .
\end{aligned}
$$

The values of $\alpha$ and $\beta$ are given in table 1. Assuming a similar $\phi$ dependence for regions $\mathrm{A}, \mathrm{B}$ and $\mathrm{D}$ as was found in region $\mathrm{C}$, the average cross section $\bar{f}$ is well approximated by the $\phi$ independent cross section

$$
\bar{f} \approx f_{\mathbf{u}}+\epsilon f_{\mathrm{L}} \text {. }
$$

Thus all regions were combined in the following studies.

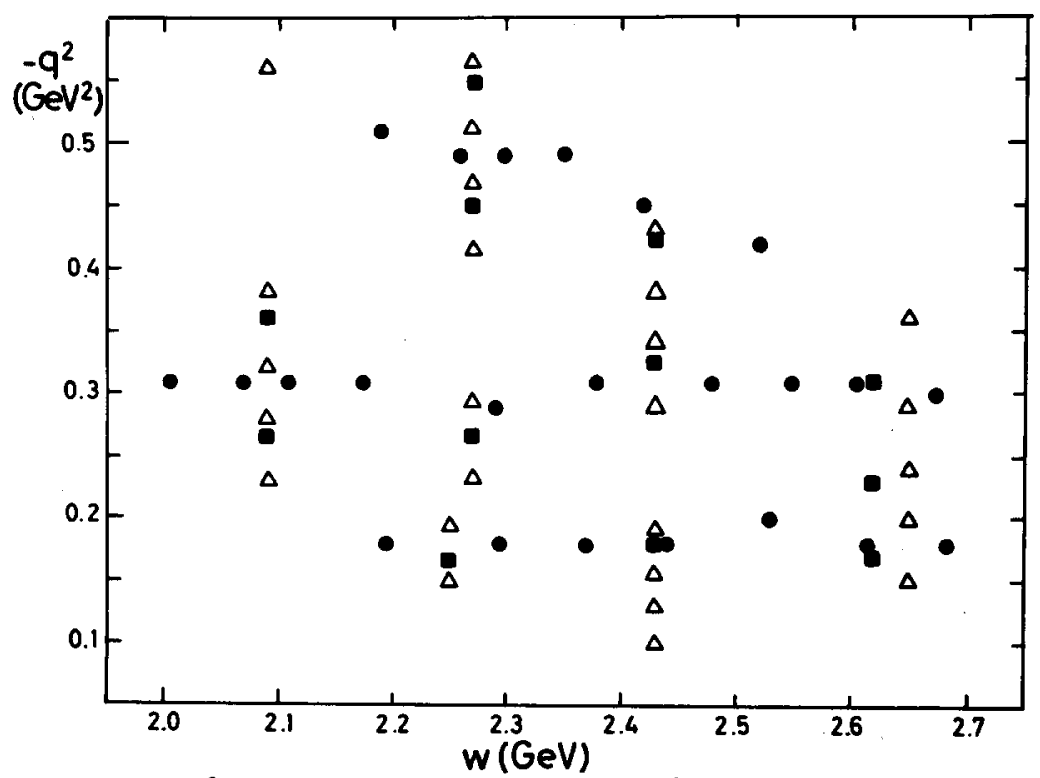

Fig. 2. Points in the $q^{2}-W$ plane used for the study of the $q^{2}$ dependence of the cross section ( $\left.\Delta\right)$; of the $W$-dependence of the cross section $(\bullet)$; of the $x$ and $p_{\perp}^{2}$ dependence of the cross section (•). 
Fig. 2 shows the points in the $\left(q^{2}, W\right)$ plane where the cross sections were evaluated. Each data point corresponds to a set of cross sections with different values of $x$ and $p_{\perp}^{2}$, and each symbol refers to a different kinematical variable used in studying the behaviour of the cross section *.

In the course of study it was occasionally necessary to extrapolate in one variable by a small amount, so that the dependence of the cross section could be investigated at the fixed values of the remaining variables. The following function was used for this purpose:

$$
F=W^{(0.27-9.42 x)} \mathrm{e}^{\left(8.13 x-3.86 x^{2}-2.5 p_{\perp}^{2}\right)},
$$

which was obtained in an overall fit to the data (the coefficient of $p_{\perp}^{2}$ was kept fixed). The cross sections were never altered by more than $15 \%$ through the extrapolation and in most cases the change was much smaller. The same function was also used to extrapolate the data from other experiments for comparison with our data.
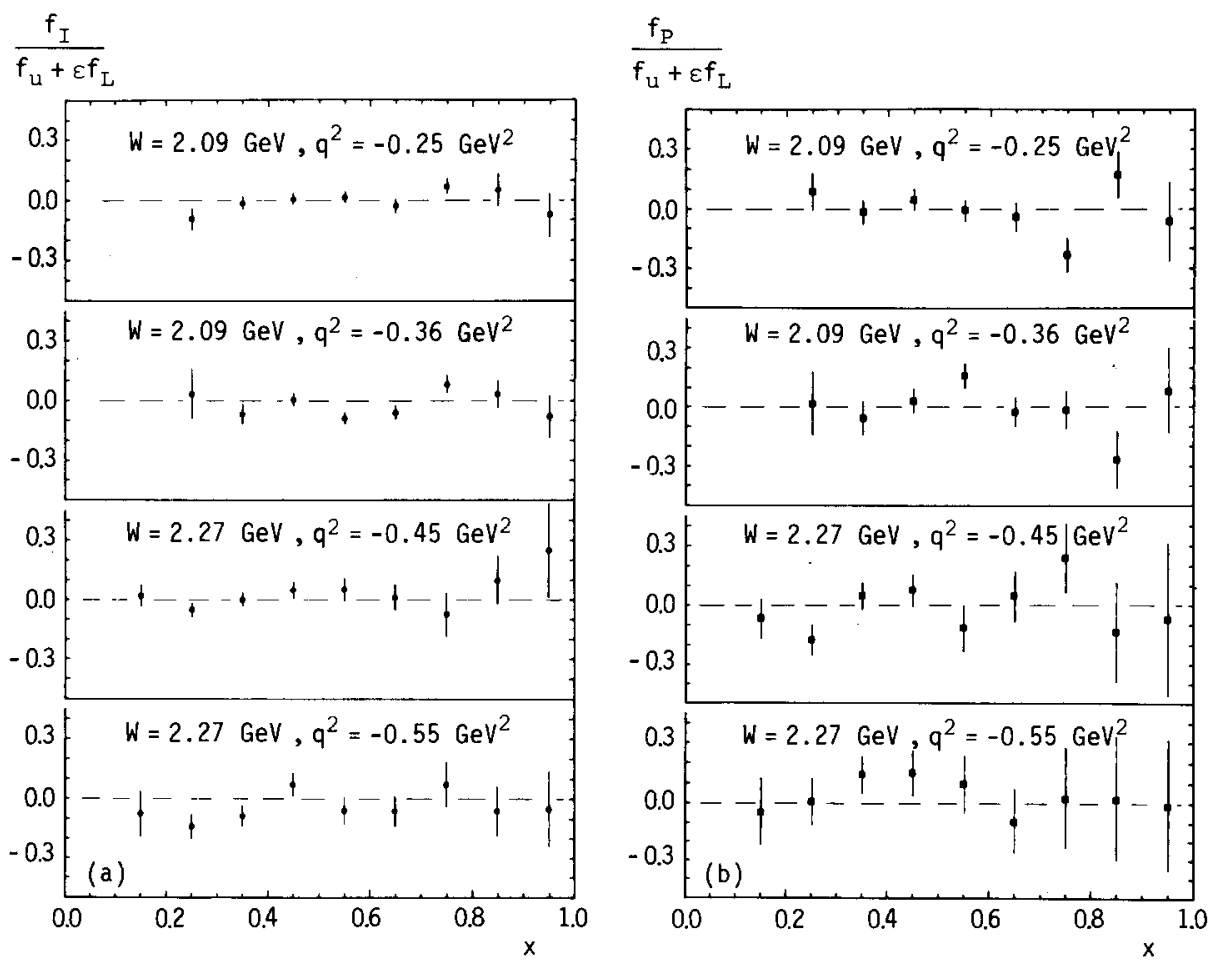

Fig. 3. $x$-distributions for (a) $f_{\mathrm{l}} /\left(f_{\mathrm{u}}+\epsilon f_{\mathrm{L}}\right)$; (b) $f_{\mathrm{p}} /\left(f_{\mathrm{u}}+\epsilon f_{\mathrm{L}}\right)$.

* A table of the measured cross sections can be found in DESY $76 / 44$. 

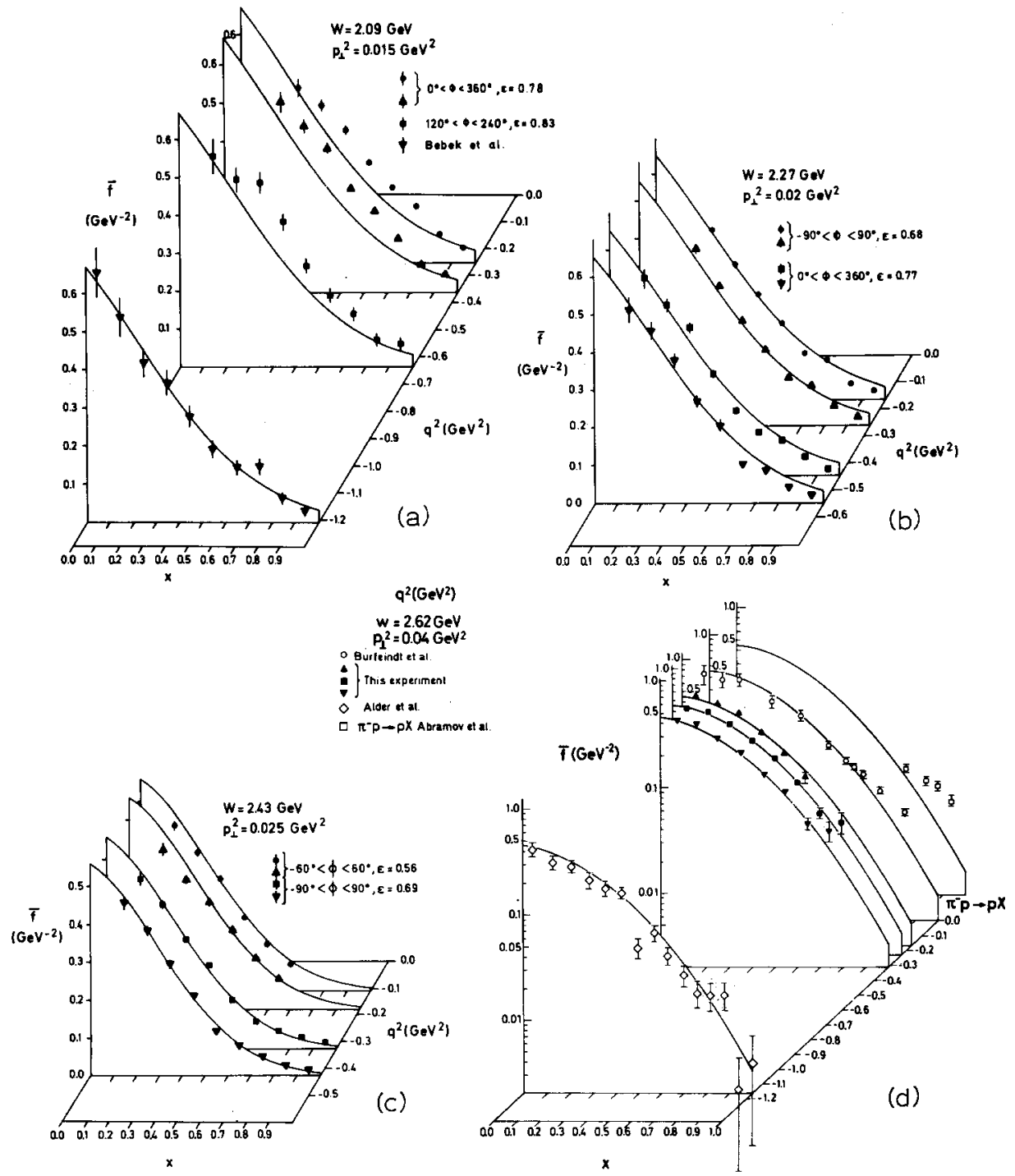

Fig. 4. $x$-distributions for $\bar{f}$. (a) $w=2.09 \mathrm{GeV}$. (b) $w=2.27 \mathrm{GeV}$. (c) $w=2.43 \mathrm{GeV}$. (d) $w=$ $2.62 \mathrm{GeV}$. The solid lines are for guidance of the eye and have the same exponential form for each value of $W$.

\section{Longitudinal momentum distributions}

The dependence of the invariant cross section on $x$ was investigated at 14 values of $W$ and $q^{2}$ (fig. 2). The data points were chosen such that several values of $q^{2}$ cor- 
responded to a fixed, or almost fixed, value of $w$, hence allowing one to study the $q^{2}$ dependence of the $x$ distributions (figs. $3 a, b$ and $4 a-d$ ). The data obtained in this experiment can be fitted well to the form $e^{c+d x+e x^{2}}$. Except for lowest $W$-region this form is independent of the $q^{2}$ at fixed values of $W$ and $p_{1}^{2}$. At $W=2.09$ $\mathrm{GeV}$ the data are shown in fig. 4 a together with the results of an electroproduction experiment [7] at higher $q^{2}$. While at higher values of $x$ no dependence on $q^{2}$ is observed, with decreasing $x$ the invariant cross section exhibits systematic deviation from $q^{2}$ independent form. In fig. $4 \mathrm{~d}$ the photoproduction data of ref. [8] and the electroproduction data of ref. [10] are compared with our data at $W=2.62 \mathrm{GeV}$. Except for some $10 \%$ difference among the different experiments at very low values of $x$ no dependence on $q^{2}$ is observed. For comparison the cross sections from a totally different reaction namely $\pi^{-}+p \rightarrow p+X$ (ref. [11]) are plotted in the same picture. They agree remarkably well with the photo- and electroproduction data. The outstanding feature in the above data is that the normalized cross section decreases by an order of magnitude along the $x$-scale. The fall is the faster the higher the $W$ is, a feature which manifests itself in the $W$-dependence of the cross section discussed later on.

The cross sections $f_{\mathrm{p}}$ and $f_{\mathrm{I}}$ normalized to $f_{\mathrm{u}}+\epsilon f_{\mathrm{L}}$ are plotted in figs. $3 \mathrm{a}$, b. They are all consistent with zero and no structure in $x$ is apparent.
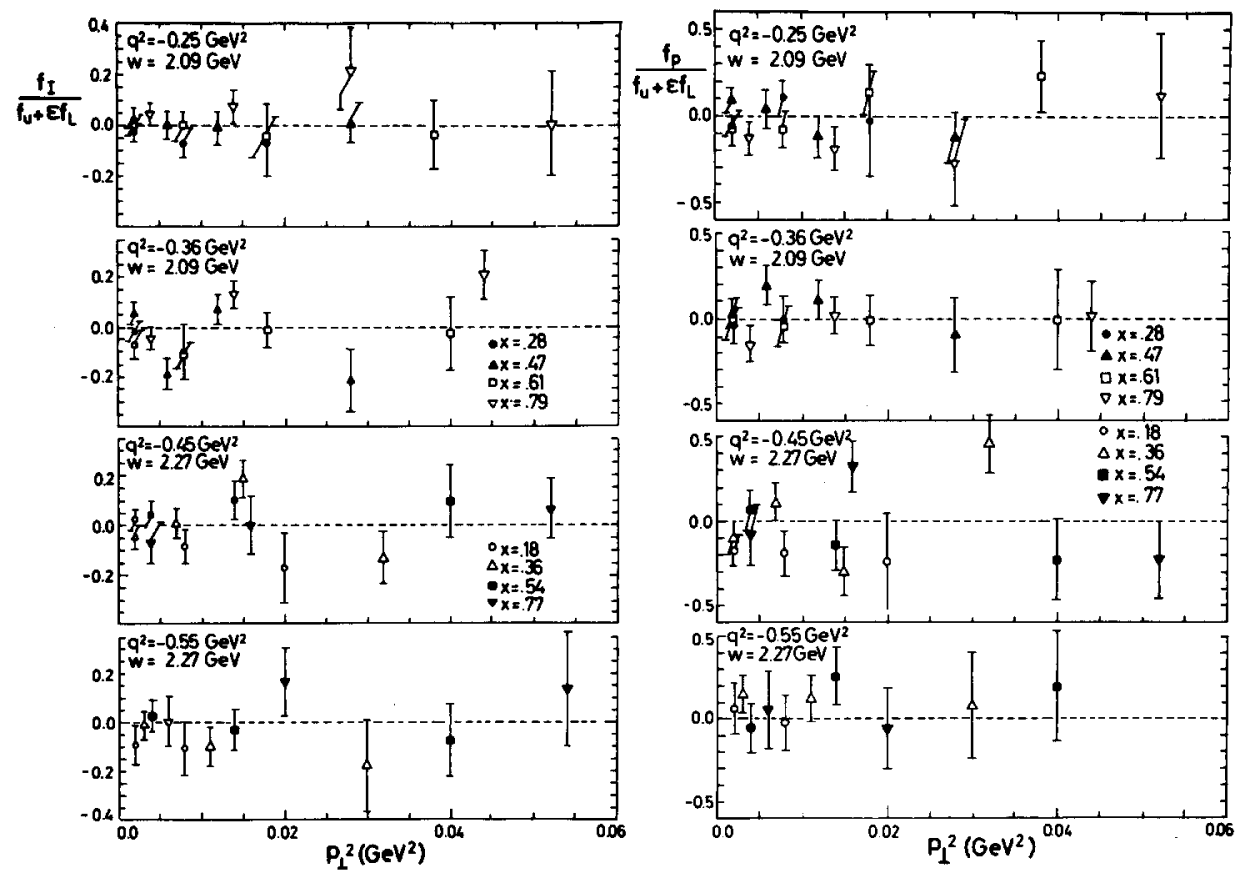

Iiig. 5. $p_{\perp}^{2}$ dependence of (a) $f_{\mathrm{I}} /\left(f_{\mathrm{u}}+\epsilon f_{\mathrm{L}}\right) ;\left(\right.$ b) $f_{\mathrm{p}} /\left(f_{\mathrm{u}}+\epsilon f_{\mathrm{L}}\right)$. 


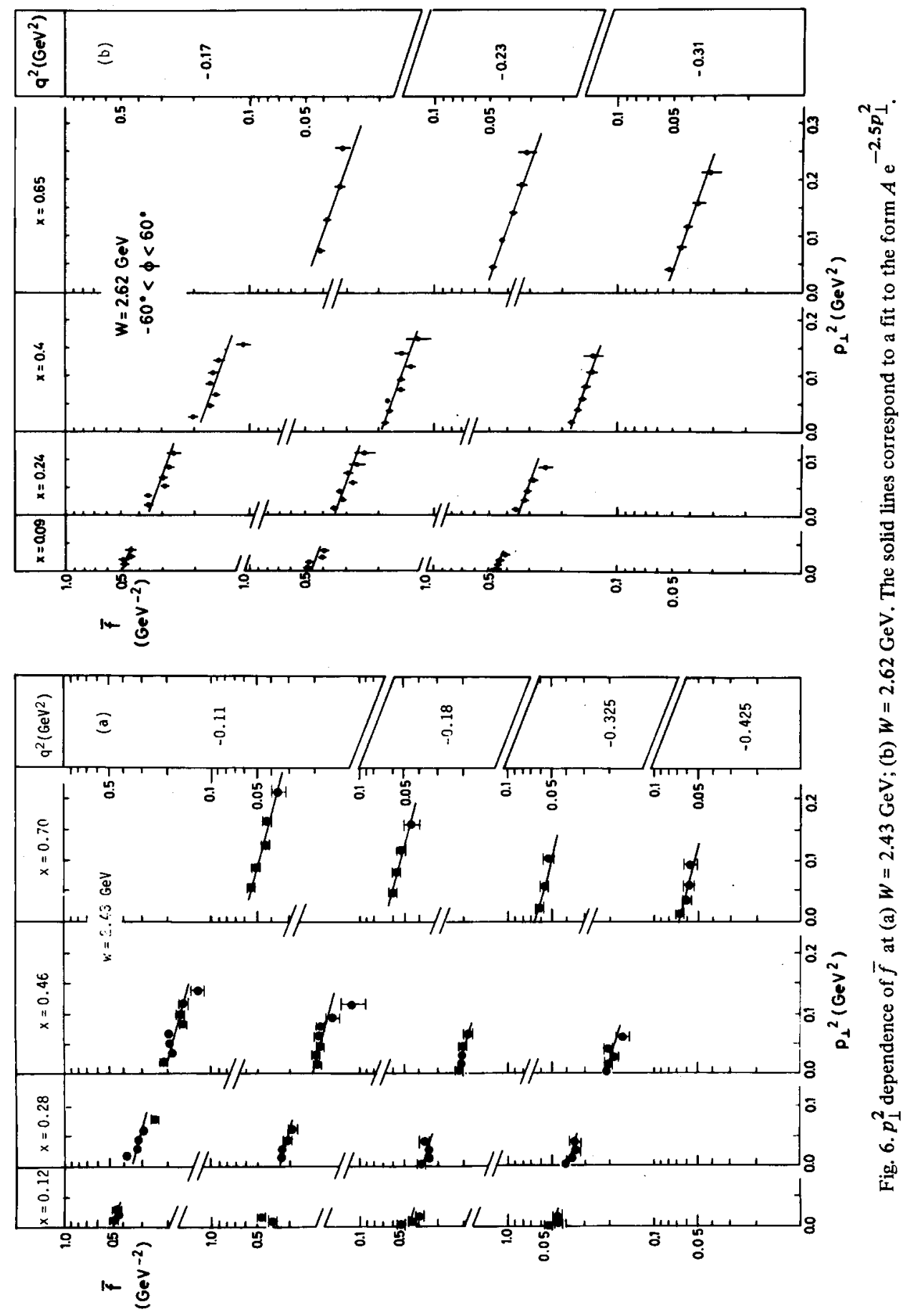




\section{Transverse momentum distributions}

The dependence of the invariant cross section on $p_{\perp}^{2}$ was studied for different values of $x$ at $q^{2}-W$ points mentioned in sect. 4 (figs. 5a, $\mathrm{b}$ and $6 \mathrm{a}, \mathrm{b}$ ). The cross section decreases with increasing $p_{\perp}^{2}$ and the distributions can be approximated by
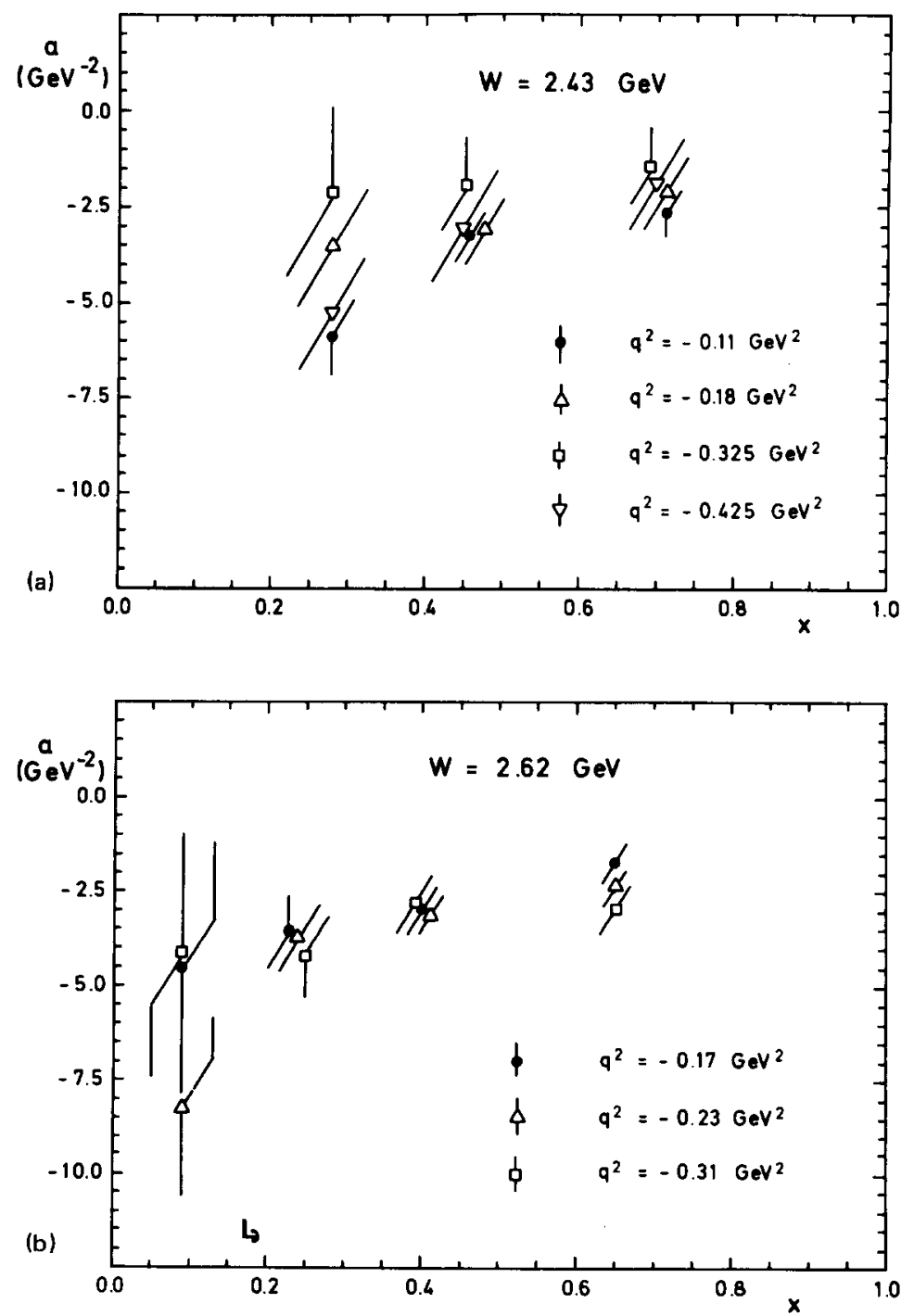

Fig. 7. The variation with $x$ of the slope $a$ in $A \mathrm{e}^{a p_{\perp}^{2}}$ for different values of $q^{2}$ at (a) $W=2.43$ $\mathrm{GeV}$; (b) $w=2.62 \mathrm{GeV}$. 


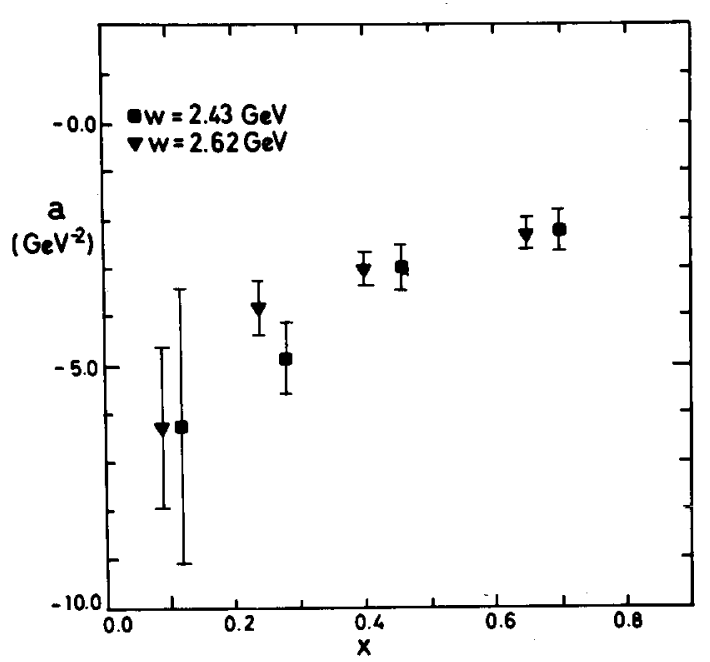

Fig. 8. The slope $a$ in $A \mathrm{e}^{a p_{\perp}^{2}}$ averaged over $q^{2}$ depicted as a function of $x$.

the form $A \mathrm{e}^{a p_{\perp}^{2}}$. No systematic variation of the slope $a$, within the limited range of $q^{2}$ is apparent. As an example the values of $a$ for different values of $q^{2}$ at the c.m. energies $W=2.43$ and $2.62 \mathrm{GeV}$ are shown in figs. $7 \mathrm{a}, \mathrm{b}$. To investigate the $x$ dependence of $a$, the data were averaged over $q^{2}$. The results for the above values of $W$ are shown in fig. 8 where a continuous decrease of $|a|$ with increasing $x$ is observed. This structure is similar to the one observed in the electroproduction of $\pi^{-}$ at comparable c.m. energies [12] and purely hadronic reactions $\pi^{ \pm} p \rightarrow \pi^{ \pm} X$ at higher values of $W[13]$, where in both experiments the average transverse momentum, which for a normal distribution is inversely proportional to $\sqrt{|a|}$, shows a maximum at medium values of $x$. The slope $a$ has also been measured in a photoproduction experiment $\gamma \mathrm{p} \rightarrow \mathrm{p}+\mathrm{X}$, but at a higher c.m. energy $W=3.5 \mathrm{GeV}[14]$. There only a slight decrease of $|a|$ in the range $x=0.2$ to 0.6 was observed. It must, however, be noted that the photoproduction and the present experiment use, specially at lower values of $x$, different portions of the $p_{\perp}^{2}$ distributions for the calculation of the above slope.

As in the longitudinal momentum distributions, the transverse polarization and interference terms show no dependence on $p_{\perp}^{2}$ and within the error bars they are consistent with zero (figs. 5a, b).

\section{6. $W$ dependence}

The dependence of the invariant cross section on the c.m. energy, $W$, was studied at the different values of $q^{2}$ which were subdivided into three or four $x$ regions (figs. $9 \mathrm{a}-\mathrm{c})$. In the observed range of $W$, the cross section falls monotonically with in- 

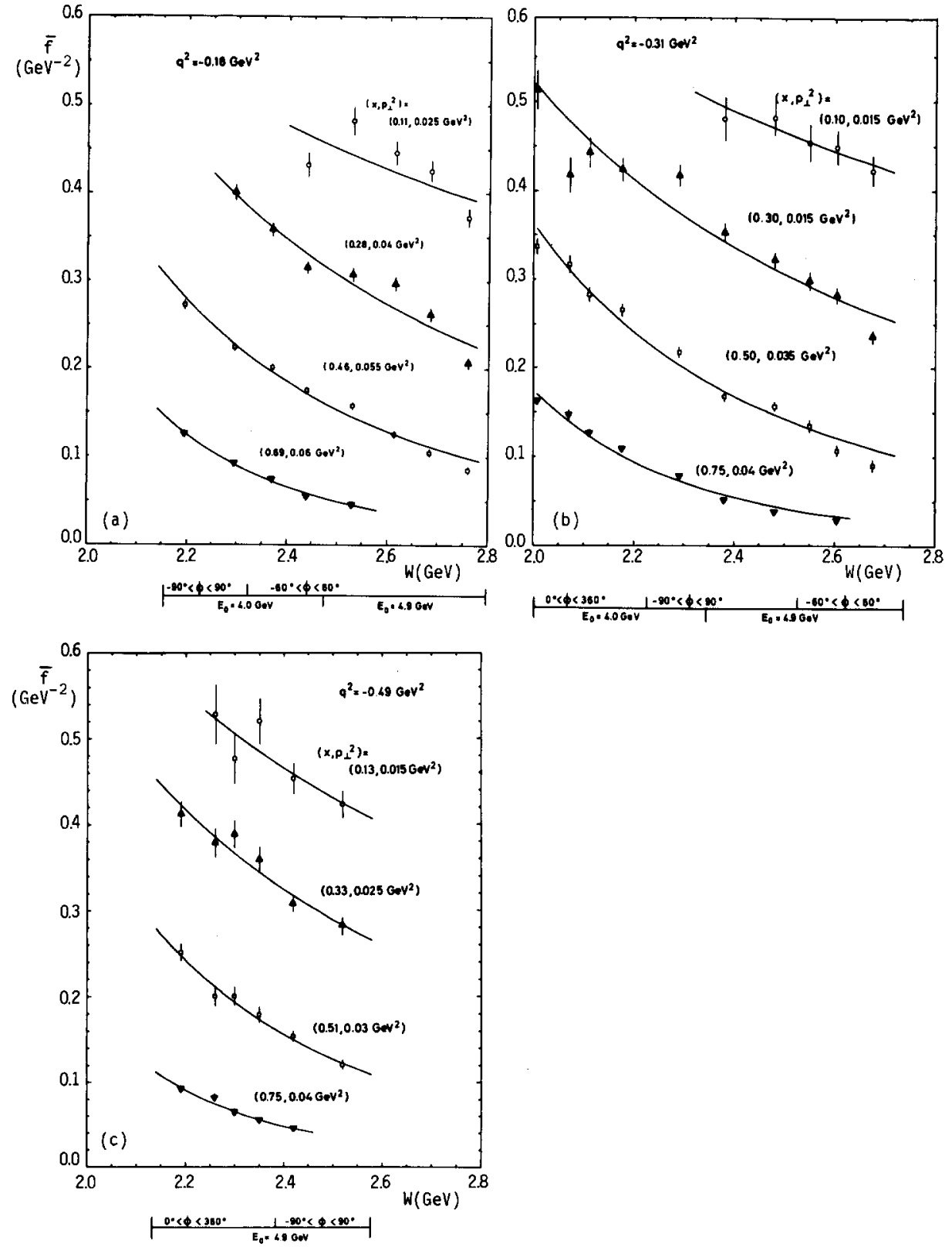

Fig. 9. $W$-dependence of $\bar{f}$ for (a) $q^{2}=-0.18 \mathrm{GeV}^{2}$; (b) $q^{2}=-0.31 \mathrm{GeV}^{2}$; (c) $q^{2}=-0.49 \mathrm{GeV}^{2}$. The solid lines correspond to a fit to the form $A\left(W^{2^{2}}-M_{\mathrm{p}}^{2}\right)^{b}$. 


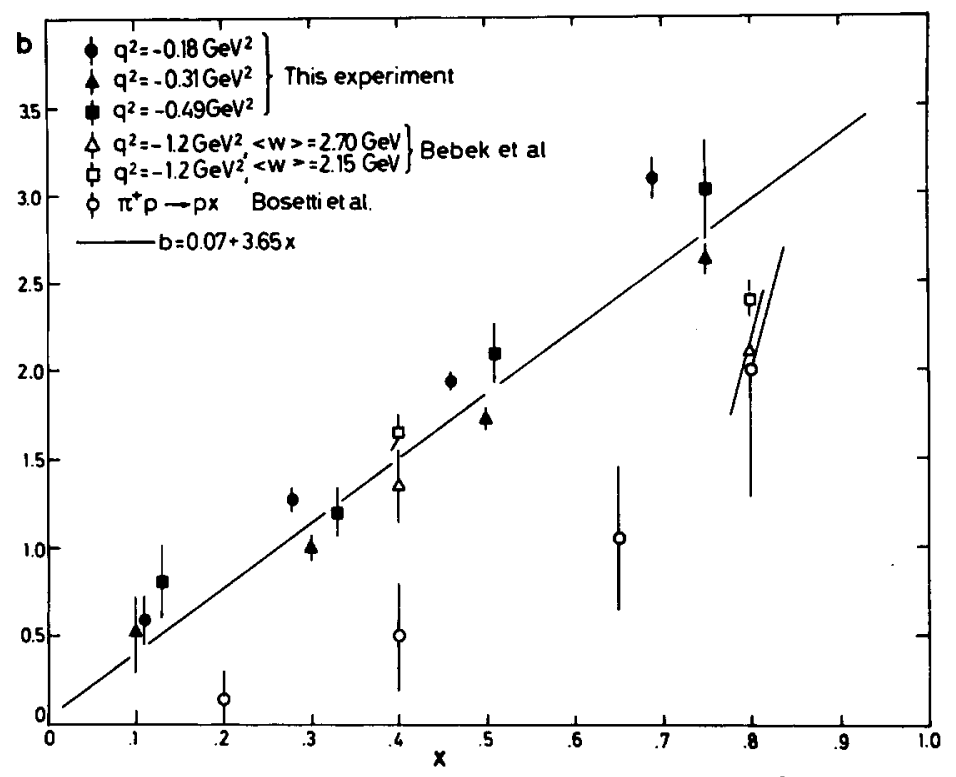

Fig. 10. The dependence of the power $b$ in $A\left(W^{2}-M_{\mathrm{p}}^{2}\right)^{b}$ on $x$.

creasing $W$ and in nearly all cases it is well represented by the form $A\left(W^{2}-M_{\mathrm{p}}^{2}\right)^{-b}$. Whereas the power $b$ does not show strong dependence on $q^{2}$ it increases linearly with increasing $x$ (fig. 10). Only for $x \sim 0.5$ is the observed $W$-dependence compatible with the predictions of a simple nucleon exchange model. At lower $x$ the cross section decreases more slowly with increasing $W$ and at higher $x$ the dependence on $W$ is stronger. This observation is supported by the results of the electroproduction experiment described in ref. [7] where a similar rise in the value of the power $b$ is observed at $q^{2}=-1.2 \mathrm{GeV}^{2}$. On the other hand the comparison of the two photoproduction experiments $[7,13]$ with $W=2.62 \mathrm{GeV}$ and $W=3.5 \mathrm{GeV}$, respectively, yield a value of about 1.5 for $b$ which does not appear to depend on $x$. The fact that in the present experiment the value of $\epsilon$ changes with $W$ does not alter the picture much. Under the extreme assumptions that the cross section is purely longitudinal, the value of $b$ decreases by about 0.5 for all values of $x$, hence affecting merely the scale. The above feature is not specific to electroproduction reactions. In a purely hadronic process $\pi^{+} \mathrm{p} \rightarrow \mathrm{pX}$ a continuous increase of the power $b$ with $W$ has also been observed [13]. The results from the above experiment are also shown in fig. 10. The large error bars are due to the fact that the values of the cross section had to be extracted from a small figure, as the cross sections were not tabulated.

\section{7. $q^{2}$ dependence}

The dependence of the invariant cross section on $q^{2}$ was studied at four c.m. ener. gies in different $x$ intervals (figs. $11 \mathrm{a}-\mathrm{d}$ ). Except at lowest $W$ regions the cross sec- 


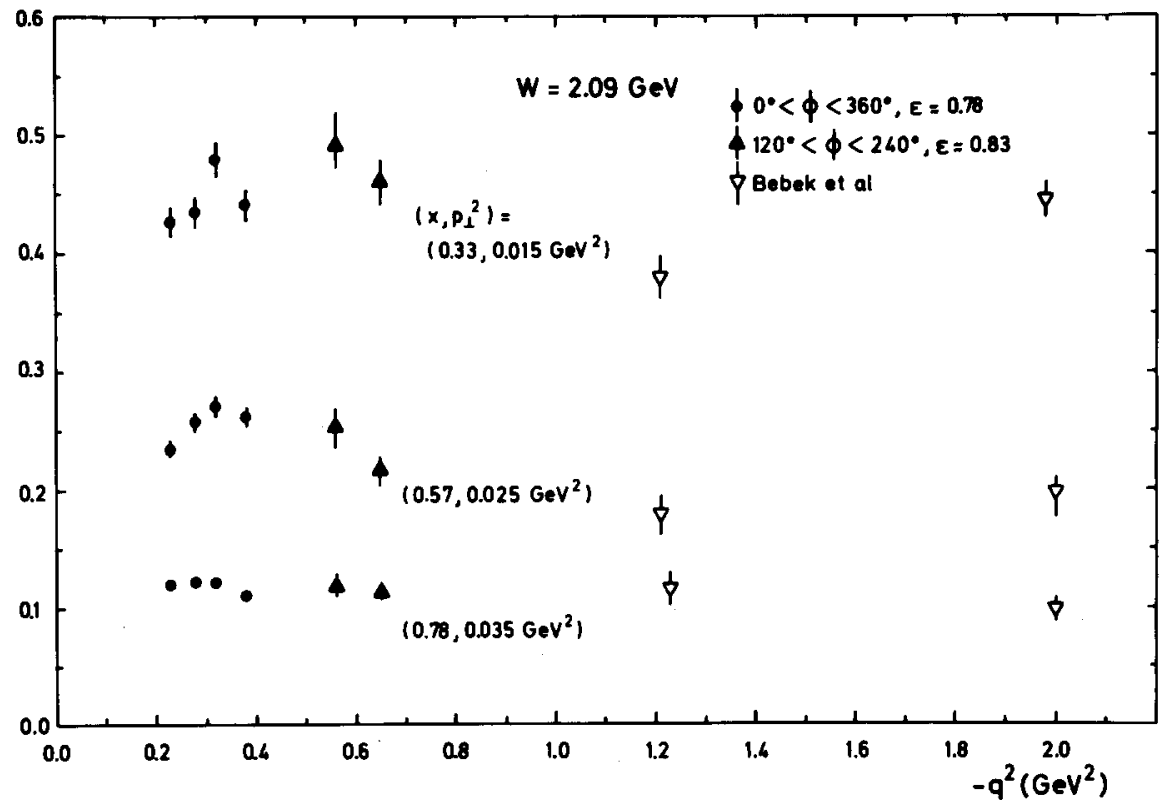

(a)

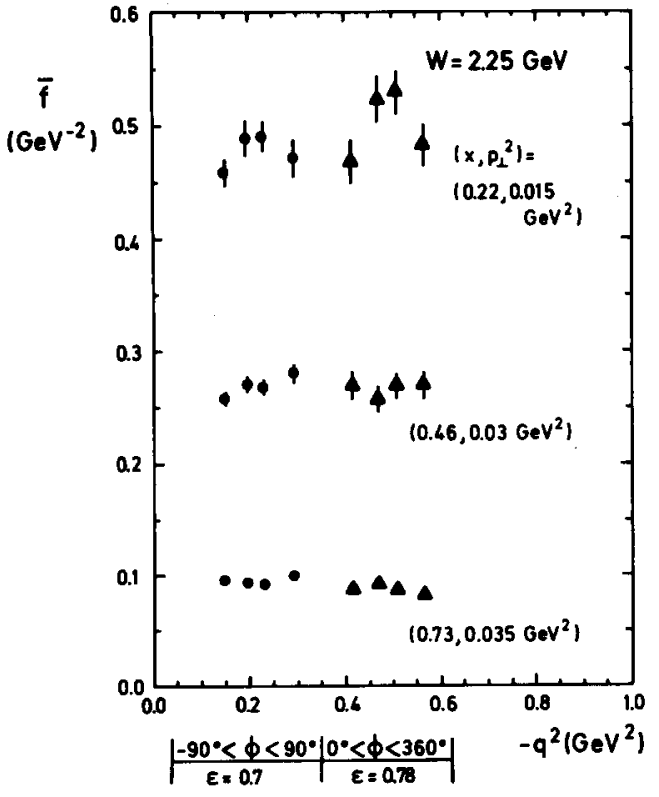

(b)

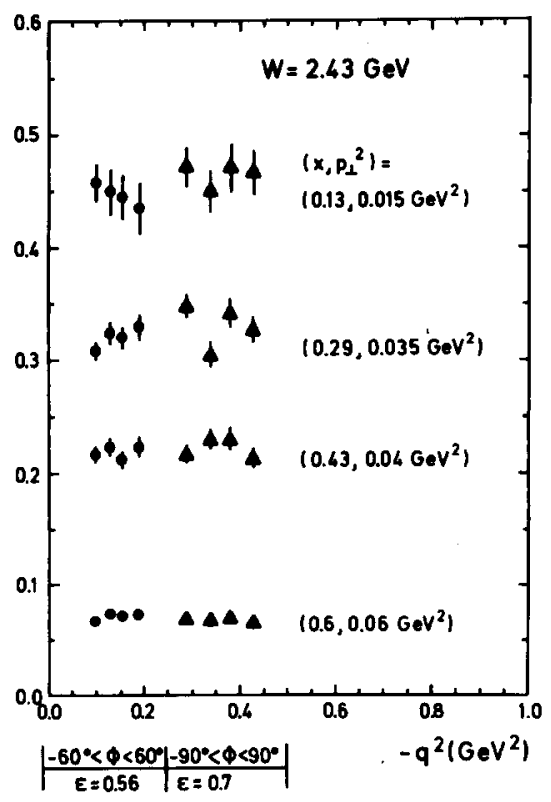

(c) 


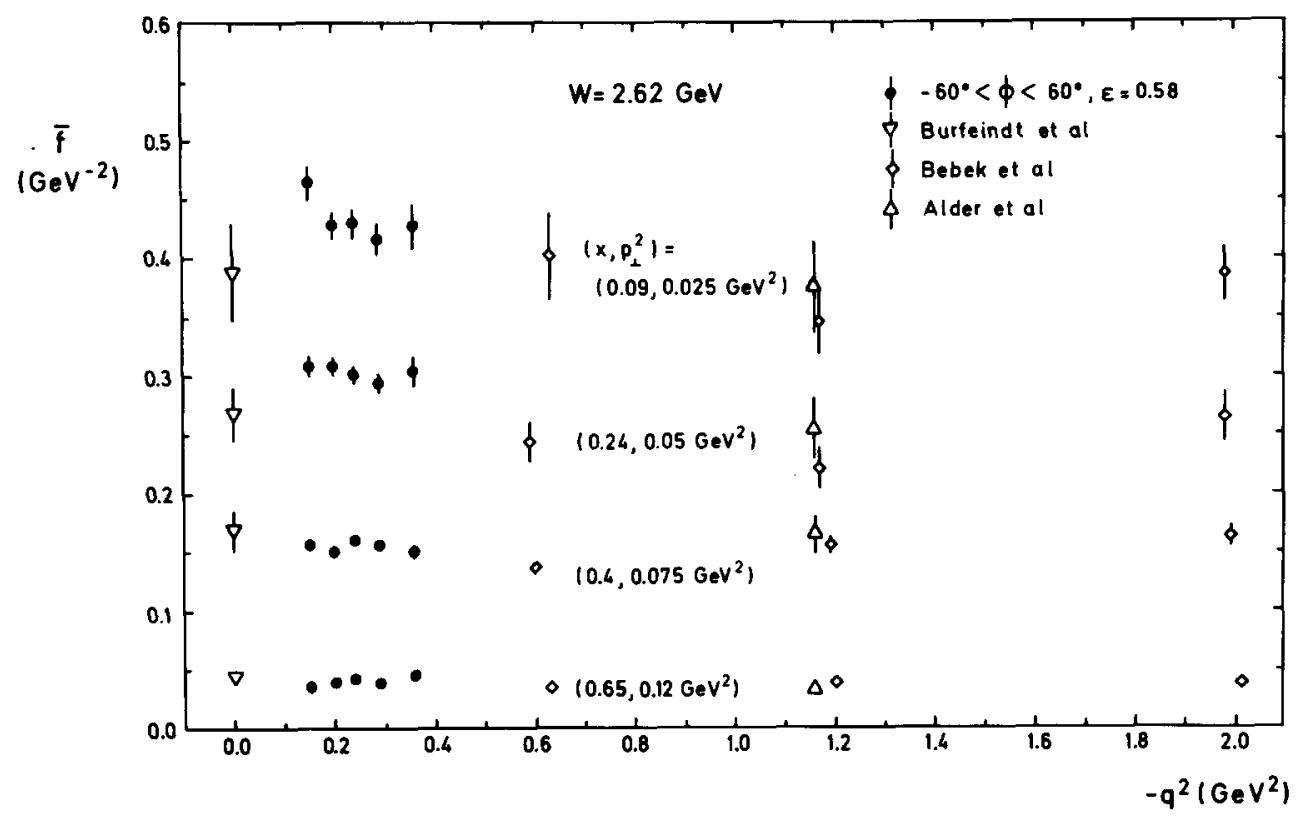

(d)

Fig. 11. The $q^{2}$ dependence of $\bar{f}$ for (a) $W=2.09 \mathrm{GeV}$; (b) $W=2.27 \mathrm{GeV}$; (c) $W=2.43 \mathrm{GeV}$; (d) $W=2.62 \mathrm{GeV}$.

tion shows little variation with $q^{2}$. At $W=2.09 \mathrm{GeV}$ and $x=0.57$ an enhancement of the magnitude of roughly $25 \%$ near $q^{2}=-0.4$ is observed. At a lower value of $x$ a less prominent structure is also visible, but at the highest value of $x$ the distributions are completely flat. Although there is some contribution to the cross section from the final state $\mathrm{p} \rho^{\mathbf{0}}$, in the kinematical interval where the enhancement is manifest, it can not explain the observed effect, since in that case one would expect a stronger effect in the regions $W=2.09 \mathrm{GeV}, x=0.78$ and $W=2.25 \mathrm{GeV}, x=0.73$ due to larger contributions from the above channel. Where it was possible the cross sections were compared with the photoproduction values [8] and the electroproduction data at higher $q^{2}[7,9,10]$. At $W=2.09 \mathrm{GeV}$ and $x=0.33$ and 0.57 the higher $q^{2}$ data are consistent with the levelling off of the structures mentioned above. In the other regions there is no deviation from constant behaviour except that at very low values of $x$ at $W=2.62 \mathrm{GeV}$ our data have slightly higher values.

\section{Summary}

(i) The invariant cross section does not significantly depend on the azimuthal angle $\phi$, i.e. $f_{\mathrm{p}}$ and $f_{\mathrm{I}}$ are small compared with $f_{\mathrm{u}}+\epsilon f_{\mathrm{L}}$.

(ii) The invariant cross section $f$ decreases strongly with $x$. 
(iii) The $p_{\perp}^{2}$ distributions show an exponential decrease whose slope varies with $x$.

(iv) The invariant cross section decreases with increasing $W$ with an inverse power that grows linearly with $x$.

(v) No dependence of the normalized invariant cross section on $q^{2}$ is observed except at $W=2.09 \mathrm{GeV}$ and $x<0.66$ where an enhancement is detected.

Some of us (E.G., F.J., H.D.M., H.D.R. and D.S.) would like to thank Professors H. Schopper and G. Weber for the kind hospitality at DESY. We are indebted to Professor K. Heinloth and Drs. I. Dammann and C. Driver for their valuable assistance during the data collection. We also want to thank Mr. G. Augustinski, Mr. P. Burmeister, Mr. K. Maschidlauskas, Mrs. B. Nissen and Mrs. R. Siemer for their excellent assistance. The valuable cooperation of the Synchrotron crew, the Hallendienst, the Kältetechnik and the Rechenzentrum is gratefully acknowledged.

\section{References}

[1] T. Azemoon et al., Contrib. no. 116 to Int. Symp. on electron and photon interactions at high energies, Bonn, 1973.

[2] L.N. Hand, Phys. Rev. 129 (1963) 1834;

S.M. Berman, Phys. Rev. 135 (1964) 1249.

[3] F.W. Brasse et al., Nucl. Phys. B46 (1972) 415.

[4] C. Driver et al., Nucl. Phys. B30 (1971) 245.

[5] T. Azemoon et al., Nucl. Phys. B95 (1975) 77.

[6] H. Ackermann, Thesis, University of Marburg 1976.

[7] C.J. Bebek et al., Phys. Rev. Letters 34 (1975) 1115.

[8] H. Burfeindt et al., Phys. Letters 43B (1972) 345.

[9] C.J. Bebek et al., Phys. Rev. Letters 32 (1974) 27.

[10] J.C. Alder et al., Nucl. Phys. B46 (1972) 415.

[11] B.M. Abramov et al., Preprint Moscow. Inst. for Theoretical and Experimental Physics GKAE-ITEF-35 (1973).

[12] DESY-Glasgow Collaboration, Contrib. no. 242 to 7 th lepton-photon Symp., Stanford, 1975.

[13] P. Bosetti et al., Nucl. Phys. B54 (1973) 141.

[14] H. Burfeindt et al., Nucl. Phys. B74 (1974) 189. 